\title{
Development and applications of THz gyrotrons
}

\author{
M.Yu. Glyavin \\ Institute of Applied Physics of Russian academy of Sciences, Nizhny Novgorod, Russia, glyavin@appl.sci-nnov.ru
}

\section{Introduction}

The last decade has contributed to the rapid progress in the development of $\mathrm{THz}$ sources in particular gyrotrons. The CW gyrotron developed at IAP RAS provides radiation with a power up to $1 \mathrm{~kW}$ in the frequency range 0.26-0.52 THz and the gyrotrons with pulsed magnetic fields operates with $100 \mathrm{~kW}$ power level at $0.7 \mathrm{THz}$ and 1 $\mathrm{kW}$ at $1 \mathrm{THz}$ frequency.

\section{Results}

The main results of IAP RAS activity with $\mathrm{THz}$ band gyrotron development are summarized below.

A series of experiments on application of CW THzradiation for spectroscopy, nano-powders manufacturing, and gas discharge initiation has been carried out [1-3]. The possibility of achieving of unique results has been demonstrated. For example, the increase of the sensitivity of a radio-acoustic detector by several orders of magnitude, several-times increase in powders formation speed has been demonstrated. For the first time, a continuous (tens of seconds) gas discharge with a characteristic size of $1 \mathrm{~mm}$ localized in a gas stream has been demonstrated. The duration of the discharge was limited only by overheating of quasi-optical elements of the transmission line.

A physical model of accounting for the ion compensation of the space charge of the electron beam in the transporting channel of the gyrotron, including the cavity region, has been developed. An analytic solution of the ion compensation problem in a long-pulse regime have been derived. An equilibrium concentration of ions in the gyrotron's operating space after the transient processes and the profile of the electron concentration in the transporting channel have been determined. These results allow one to estimate the change of the pitch-factor due to a partial compensation of potential degradation in the gyrotron's cavity and, thus, are important for starting currents, efficiency and mode competition analysis [4].

For solution of the mode selection problem in gyrotrons at the cyclotron harmonics, a scheme, based of decreasing of diffraction quality factors of quasi-cut-off parasitic modes through their transformation into other, traveling modes was suggested. The cavity of the gyrotron in the simplest case has the form of a quasi-regular systems with a single irregularity (local radius increase) situated at the center of the cavity. While the radius of the regular parts of the cavity corresponds to the cut-off of the operating mode being excited at the second cyclotron harmonics, the local increase of the radius corresponds to the cut-off of the same wave, but with an additional radial variation. Numerical simulation of the electrodynamic cavity of a $1.3 \mathrm{THz}$ gyrotron at the second cyclotron harmonic has been preformed. Owing to the profile of the cavity, the starting current at the second cyclotron har- monic (about $10 \mathrm{~A}$ ) becomes substantially lower than the currents of the parasitic waves at the fundamental harmonic (20-30A). These results make it attractive to experimentally realize gyrotrons with unique parameters: selective excitation of the $2^{\text {nd }}$ harmonic, power of tens of $\mathrm{kW}$ and frequency of $1.3 \mathrm{THz}$.

An experimental investigation of a pulsed gyrotron with an axis-encircling electron beam $(80 \mathrm{kV} / 0.7 \mathrm{~A})$ with a sectioned klystron-like cavity, operating at the third cyclotron harmonic at a frequency of about $1 \mathrm{THz}$ has been carried out. A sectioned klystron-like cavity with a long wave-beam interaction space and a relatively low diffraction Q-factor has been suggested.

The scheme of a gyrotron with a planar configuration of the interaction space and sheet electron beam proposed by the authors of the project is of interest for the generation of sub-millimeter radiation at the harmonics of the gyro-frequency. Through the PIC simulation by the CST STUDIO SUITE code, the ability to effectively generate the 3rd harmonic in a planar gyrotron with an operating frequency of about $250 \mathrm{GHz}$ has been demonstrated. The gyrotron was fed by a sheet electron beam with an energy of $80 \mathrm{keV}$, a current of $15 \mathrm{~A}$ and the pitch factor $\sim 1$. In simulations, the steady state regime establishment at the 3rd cyclotron harmonic generation was observed at the guiding magnetic field of about $3.35 \mathrm{~T}$. Generation efficiency at these settings was $8 \%$ and the output power $\sim 50 \mathrm{~kW}$. Increasing of the power level up to $0.3 \mathrm{MW}$ while maintaining a selective excitation of the operating mode at the third harmonic in this circuit can be achieved by increasing the cross-section of the system up to $10 \mathrm{~cm}$ (i.e., up to 100 wavelengths) with a corresponding increase in the total beam current.

The THz band pulsed gyrotron has been used successfully for initiation of gas discharges [5]. Such plasma is promising for development of a point source of multicharged ions, a source of high-energy ultraviolet (extreme ultraviolet EUV or XUV) [6] and remote detection of remote detection of concealed radioactive materials [7]. The discharge developed and was localized only in a small region of space (about $1 \mathrm{~mm}$ ) near the gas injection hole, where the pressure was high (close to the atmospheric one) and the breakdown conditions (the "pointlike" discharge) were fulfilled. The density of electrons in the discharge was measured by observing the Stark broadening of the atomic radiation line $\mathrm{H}_{\alpha}$ of hydrogen (656.3 $\mathrm{nm}$ ) which was present in the discharge as a minor admixture. The plasma density in the discharge was equal to about $2 \cdot 10^{16} \mathrm{~cm}^{-3}$, which exceeds the cut-off density for a frequency of $0.67 \mathrm{THz}$ used to maintain the discharge. The localized gas discharge has been successfully obtained also with $1 \mathrm{~kW} / \mathrm{CW} / 0.26 \mathrm{THz}$ gyrotron.

The same gyrotron has been used also for the highresolution molecular spectroscopy in a gas mixture. As a result of analysis of experimental profiles of known lines 
of the rotational $\mathrm{SO}_{2}$ spectrum, it was demonstrated for the first time that an increase of radiation power by about three orders of magnitude leads to a proportional increase of the RAD spectrometer sensitivity. This permitted, in particular, to observe the weak transitions predicted earlier in the $\mathrm{SO}_{2}$ molecule.

The phase lock loop control of anode voltage has been used for frequency stabilization of $0.26 \mathrm{THz}$ tube. The width of the frequency spectrum was decreased to $1 \mathrm{~Hz}$ measured at intermediate frequency IF $=350 \mathrm{MHz}$, which corresponds $\Delta f / f=3 \cdot 10^{-12}$ with measurement time of a few seconds. The long-term stability was defined by reference clock $\left(\delta f / f \sim 10^{-9}\right.$ for quartz clock and up to $\delta f / f \sim 10^{-12}$ for rubidium clock). Obtained spectrum width and frequency stability were previously achieved in backward-wave oscillators, utilized in spectroscopy, but with power levels of tens of $\mathrm{mW}$, while stabilization system with anode voltage control in gyrotrons has no apparent limiting factors in the field of gyrotron output power - as it was demonstrated with output power of $40 \mathrm{~W}$ [8].

The last interesting feature is high speed production of pure nanopowders by material evaporation and condensation. In the case of evaporation by $1 \mathrm{~kW} / \mathrm{CW} / 0.26 \mathrm{THz}$ radiation, the evaporation rate was increased approximately in order in comparison with traditional heating methods.

For the future progress of gyrodevices we proposed tube with improved selection based on multi-beam scheme or non-uniform cavity profile. The optimization of the operational parameters and the experimental setup are in progress now with dual beam $0.79 \mathrm{THz} \mathrm{CW}$ gyrotron. The generation of series fundamental harmonic modes in the wide frequency range $(0.2-0.4 \mathrm{THz})$ with output power of the order of $100 \mathrm{~W}$ is obtained and second harmonic operation with frequency closed to design one and output power about $5 \mathrm{~W}$ has been observed. Next step of this project will design $1 \mathrm{THz}$ third harmonic gyrotron with relatively low operating voltage [9-11]. The possibility of energy recovery was investigated to grove up operation efficiency, same as was demonstrated, for example in [12].

For the excitation second harmonics at tube with pulsed coil and, correspondently, the possibility of 1.3 THz generation the scheme based on the cavity with a phase corrector is under development. The preliminary calculation shown, that the starting currents of fundamental harmonic parasitic modes can be increased in an order in contrast with traditional cylindrical cavity.

\section{Conclusion}

Thus, the series of powerful $\mathrm{THz}$ gyrotrons has been developed and successfully tested at the IAP RAS. The recent progress in the $\mathrm{THz}$ gyrotrons development opened a number of novel applications in the high-power terahertz science and technology.

\section{Acknowledgements}

The work supported by the Russian Science Foundation under the project No. 14-12-00887.

\section{References}

1. M.Yu. Glyavin, T. Idehara, S.P. Sabchevski. Development of THz gyrotrons at IAP RAS and FIR UF and their applications in physical research and high-power $\mathrm{THz}$ technologies // IEEE Trans. on Terahertz Science and Technology, 2015, V.5, No.5, P.788-797

2. M. Glyavin, G. Denisov, V. Zapevalov, M. Koshelev, M. Tretyakov, A. Tsvetkov. High-power terahertz sources for spectroscopy and material diagnostics // Physics-Uspekhi, 2016, V.59, No.6, P.595-604

3. M.Yu. Glyavin, A.V. Chirkov, G.G. Denisov et al. Experimental tests of $263 \mathrm{GHz}$ gyrotron for spectroscopy applications and diagnostic of various media // Rev. Sci. Instr., 2015, V.86, No.5, P.054705

4. A.P. Fokin, M.Yu. Glyavin and G.S. Nusinovich. Effect of ion compensation of the beam space charge on gyrotron operation // Phys. of Plasmas, 2015, V.22, No.4, P.043119

5. M.Yu. Glyavin, A.G. Luchinin, A.A. Bogdashov et al. Experimental Study of the Pulsed Terahertz Gyrotron with Record-Breaking Power and Efficiency Parameters // Radioph. and Quant. Electr., 2014, V.56, No.8-9, P.497-507

6. A.V. Vodopyanov, M.Yu. Glyavin, S.V. Golubev et al. Plasma density in discharge sustained in inhomogeneous gas flow by high-power radiation in the terahertz frequency range // Technical Physics Letters, 2017, V.43, No.2, P.186-189

7. G.S. Nusinovich, P. Sprangle, V.E. Semenov, D.S. Dorozhkina and M.Yu Glyavin. On the sensitivity of THz gyrotron based systems for remote detection of concealed radioactive materials // Journal of Applied Physics, 2012, V.111, P.124912

8. G.G. Denisov, A.P. Fokin, M.Yu. Glyavin et al. High precision frequency stabilization of a $100 \mathrm{~W} / 263 \mathrm{GHz}$ continuous wave gyrotron // 18th International Vacuum Electronics Conference 24 - 26 April 2017, London

9. N.S. Ginzburg, M.Yu. Glyavin, A.M. Malkin et al. Improvement of operation stability at high cyclotron harmonics in the double-beam $\mathrm{THz}$ range gyrotrons // IEEE Trans. On Plasma Science, 2016, V.44, No.8, P.1303-1309

10. M.Yu. Glyavin, N.A. Zavolskiy, A.S. Sedov and G.S. Nusinovich. Low-Voltage Gyrotrons // Phys. of Plasmas, 20, 033103 (2013)

11. N.S. Ginzburg, M.Y. Glyavin, I.V. Zotova, I.V. Zheleznov, A.P. Fokin. Optimization of terahertz range gyrotron self-excitation conditions by increasing the lifetime of cyclotron oscillators in low-voltage interaction space // Tech. Phys. Lett., 2017, V.43, No.1, P.110-113

12. M. Glyavin, A. Kuftin, N. Venediktov, V. Zapevalov. Experimental investigation of a $110 \mathrm{GHz} / 1 \mathrm{MW}$ gyrotron with the one-step depressed collector // Int. J. of Infrared and Millimeter Waves, 1997, V.18, No.11, P.2129-2136 